\title{
Rites et rythmes de l'eau et du désir dans Mossane de Safi Faye
}

\author{
Pujante-González, Domingo
}

Universitat de València, Domingo.Pujante@uv.es

\begin{abstract}
Resumen
La historia de Mossane (1996) de la realizadora senegalesa Safi Faye (1943-) se desarrolla en territorio serer, en Mbissel, entre mar y sabana, donde las tradiciones y los ritos religiosos marcan el ritmo de la vida del pueblo. La película comienza y acaba con el mismo movimiento de la cámara alrededor de la bella Mossane en el agua. El agua es, en efecto, un componente primordial de la narración fílmica pero posee un valor simbólico ambivalente, en continua transición entre la vida y la muerte. El elemento acuático se muestra como un preciado bien en esta zona de África que, sin embargo, puede ser premonitorio de un final funesto. La película está marcada por una serie de imágenes en las que el agua está muy presente: en la belleza de los paisajes de esta entrada de mar cercana al pueblo, en los rituales de los baños de las mujeres o en el pozo excavado en medio de las tierras áridas donde otras mujeres van a por ella para traerla a la aldea. En este trabajo, analizaremos esta rica presencia del agua que marca no sólo la memoria colectiva y los ritmos de la comunidad en espacios naturales sino también los rituales íntimos en los que las mujeres desnudas hablan de sus deseos y se abren a la sexualidad.
\end{abstract}

Palabras clave : ritos ; agua ; mujer ; deseo ; Safi Faye.

\section{Résumé}

L'histoire de Mossane (1996) de la réalisatrice sénégalaise Safi Faye (1943-) se déroule en pays Sérère, à Mbissel, entre mer et savane, où les traditions et les rites religieux marquent le rythme de la vie du village. Le film s'ouvre et se termine par le même mouvement de la caméra autour de la belle Mossane dans l'eau. En effet, l'eau est un composant primordial de la narration filmique mais elle possède une valeur symbolique ambivalente, toujours en transition entre la vie et la mort. L'élément aquatique est montré comme un bien précieux dans cette région africaine, pouvant, toutefois, être prémonitoire d'une fin funeste. Le film est donc ponctué d'images où l'eau est très présente: dans la beauté des paysages de cette branche de mer à proximité du village, dans les rituels des bains des femmes ou dans le puits au milieu des terres arides où d'autres femmes vont la chercher pour l'apporter au hameau. Nous analyserons cette riche présence de l'eau marquant non seulement la mémoire collective et les rythmes de la communauté dans des espaces naturels mais également les rituels intimes où les femmes nues s'ouvrent aux désirs et à la sexualité.

Mots-clés : rites ; eau ; femme ; désir ; Safi Faye.

\begin{abstract}
The story of Mossane (1996), directed by the Senegalese film producer Safi Faye, takes place in Mbissel, Serer territory, between the ocean and the savannah, where traditions and religious rites have an impact on people's way of life. The film both begins and ends with the camera moving across the water in the stunning Mossane. Water is certainly an essential component of the film's narrative, but it has an ambivalent symbolic value, in the continuous transition between life and death. In this area of Africa the water element is considered a precious property, which can be, however, the premonition of a fatal end. The story is imprinted by a series of images in which water is always present : in the landscape around the beautiful estuary near the village, in the bathing rituals of women or in the water well excavated in the middle of arid lands, where other women go to collect water to take back to their village. In this study we will analyze this rich presence of water that marks not only the collective memory and rhythms of the
\end{abstract}


community in natural areas, but also the intimate rituals where naked women talk freely about sex and their desires.

Keywords : rites ; water ; woman ; desire ; Safi Faye.

\section{Filmer en tant que femme : une lecture genrée}

Le premier cinéma « au féminin », comme celui des réalisatrices belges Agnès Varda et Chantal Akerman, était fortement imprégné des idées féministes. La femme prenait enfin la parole dans le cinéma, très souvent d'une manière impudique, pour parler de sa « différence », de son corps, de son intimité, de ses désirs, de ses relations amoureuses et familiales, de son aliénation et de sa réification par le regard de l'homme. Il s'agit d'un cinéma intellectuel, d'introspection, revendicatif, qui cherche un langage propre. En effet, le fait de poser au cinéma la question de l'identité de genre est une façon aiguë d'interroger cette pratique. L'artiste, quel que soit son genre, regarde le monde avec son expérience propre, « travaille sur des relations humaines où la séduction, l'érotisme, les fantasmes aussi, ont leur part » (Benhamou, 2007 : 4). Sa singularité est essentielle mais aussi la façon dont il/elle «sait se mettre au point de confluence des imaginaires des autres » (Benhamou, $2007: 5$ ).

Nous avons voulu dans ce travail que cette idée d’une lecture genrée de la production cinématographique traverse nos propos et savoir comment «dans toutes ces dimensions se vivait (ou non) le fait d'être une femme » (Benhamou, 2007 : 5), en l'occurrence une femme d'Afrique noire. S'il est vrai que les nouvelles générations d'artistes préfèrent que l'on parle de leur travail artistique plutôt que de leur identité ou de leur origine et que les féminismes historiques s'effacent progressivement des consciences, il n'est pas moins vrai que la perspective de genre, et les théories sur la construction mouvante et performative de l'identité sexuelle sont au centre des réflexions et des pratiques artistiques aujourd'hui, spécialement dans des domaines particulièrement propices à ce type de questionnement, comme le sont les arts visuels. Dans toutes ces réflexions, la thématique du désir et de la sexualité, historiquement liée au regard masculin, d'un côté, et à la représentation, voire à la caméra, de l'autre, ne pouvait qu'enrichir ce vif débat.

Pour approfondir cette réflexion par rapport à la «spécificité » de la réalisation filmique au féminin, nous voudrions mettre en valeur l'idée d'Estelle Bayon (2007) pour qui les cinéastes femmes disent autrement le discours des désirs de la chair à l'image. Sur ce « dire autrement le désir », la réalisatrice belge Marie Mandy a produit en 2001 un excellent film documentaire intitulé Filmer le désir (voyage à travers le cinéma des femmes) où elle donne la parole à quinze femmes cinéastes de différents pays (Catherine Breillat, Jeanne Labrune et Agnès Varda pour la France, Paule Baillargeon et Léa Pool pour le Québec, Moufida Tlatli pour la Tunisie, Safi Faye pour le Sénégal, pour ne nommer que les francophones) afin qu'elles s'expriment précisément sur cette question du regard ou du cinéma « au féminin ». À partir de la question « être une femme change-t-il pour vous votre manière de filmer le corps, l'amour, le désir et la sexualité ? » et illustrant leurs propos par des extraits de leurs films, elles réfléchissent sur la possibilité d'un langage cinématographique de femme et le désir de fantasmer et rêver une nouvelle image d'elles-mêmes. Elles abordent donc ouvertement leur propre vision de la sexualité, des tabous et des interdits, et signalent, d'une manière générale, que le fait de vivre dans un corps de femme joue son rôle dans leurs choix artistiques, dans leur travail sur le cadrage et le traitement de l'image et, bien évidemment, dans les histoires qu'elles racontent. Toutes partent de leur frustration en tant que spectatrices et le constat du manque d'authenticité dans la représentation de leurs désirs ou de l'absence du regard de la femme sur le sexe dans les films faits par les cinéastes hommes. Elles insistent sur les rapports de pouvoir étroitement liés à des questions comme le sexe et la maternité. Une autre constante est le recours, sous un autre prisme, à la sexualité, plus ou moins crue, plus ou moins voilée, selon les pays et la personnalité de chaque réalisatrice. Elles essaient toutes de rendre visible cet autre regard, un miroir plus fidèle à ce qu'elles ressentent intimement (Pujante, 2013 : 30-31). Quant à l'écriture des histoires qu'elles racontent, comme le souligne Marta Segarra, au-delà du fait que « ces réalisatrices écrivent souvent les scénarios de leurs films, ou que parfois elles se consacrent de même à l'écriture littéraire, le cinéma déploie désormais une énorme influence non seulement sur l’imaginaire des lecteurs mais aussi sur celui des écrivains » (Segarra, $2010: 8)$. 


\title{
2. Filmer en tant qu'africaine : une lecture postcoloniale
}

Olivier Barlet signale que le cinéma (africain) « essentiellement fait par des hommes passe souvent par les femmes pour interroger la virilité de la société » (Barlet, 1996 : 116). Il ajoute que, face à la supériorité, au paternalisme et à l'extériorité du regard, le premier mouvement des cinéastes africains sera « de revendiquer l'authenticité de leur regard sur leur propre réalité » (Barlet, 1996 : 18). La question du regard ethnographique, comme l'affirme le cinéaste sénégalais Joseph Gaï Ramaka dans un entretien avec Barlet, «n'est pas de savoir qui le porte mais quel est le contenu de l'ethnographie ellemême. Il faut savoir ce qu'on a envie de voir avant de savoir comment le regarder : l'Autre pour ce qu'il est et que je vais décortiquer ou bien l'Autre en ce que je me sens proche de lui et en ce qu'il est proche de moi » (Barlet, 1996 : 19). En ce sens, Safi Faye, en tant que femme noire, ethnologue et cinéaste s'intéresse moins aux archives qu’au témoignage, à l'écoute. Pour elle, les frontières entre la fiction et le documentaire restent diffuses : « La fiction est jouée mais provient tant de la vie quotidienne que de l'imaginaire. On fait une mise en scène pour respecter le temps du cinéma » (Barlet, 1997 : 8). Et elle précise : « Tout est imagination, la mienne. Pourquoi n’y aurait-il que des films à message, didactiques ou éducatifs ? Créer n'est pas un calcul. Pour moi, un film provient de l'imaginaire. Dans Mossane, les cérémonies, je les ai inventées et elles n’ont rien à voir avec mes études d'ethno » (Barlet, 1997 : 11). Ce n’est pas aussi évident qu'elle le prétend.

A propos de son film Lettre paysanne (1975), le premier long métrage tourné par une femme africaine, elle déclare : « J'étais observatrice, je n’ai fait que les écouter. Je ne bougeais pas de ma place et j’ai tout filmé comme si j'étais celle à qui l'on s'adressait » (Barlet, 1996 :19). À notre sens, cette technique perdure en quelque sorte dans le film Mossane, même si l'histoire de fiction est déjà écrite auparavant par l'auteure. La vision militante de Safi Faye serait d'affirmer une politique du quotidien "sans pour autant faire l'impasse sur les questions sociales ou économiques » (Barlet, 1996 : 22). Bien qu'elle prétende à l’universalisme, pour décoloniser les écrans, il faut «proposer au public africain une nouvelle vision de son propre espace » (Barlet, 1996 : 53). La colonisation avait signifié une dépossession de l'espace et une perte d'identité. Il s'agit de « se réapproprier le territoire pour que le public puisse s'y identifier. En lui montrant des images « de chez lui », le cinéma l’aide à retrouver son identité culturelle » (Barlet, 1996 : 53).

Ainsi, Safi Faye montre dans ses films de longs panoramiques du paysage africain mais pour s'arrêter finalement sur les personnages et leurs travaux. En ce sens, André Gardies souligne que « à la différence du cinéma occidental qui montre pour raconter, le cinéma africain me semble raconter pour montrer » (Gardies, 1987 : 27). Le récit n'est donc que " prétexte pour que l'image serve de miroir, dévoilant un espace et des comportements supposés connus et qu'il s'agit de se réapproprier » (Barlet, 1996 : 54). Comme le précise Barlet, l'espace, qu'il qualifie « d'espace-miroir », est en réalité le personnage principal du film. Le geste est certes idéologique. Or, cette volonté de filmer les réalités africaines du point de vue de la femme ne fait pas du cinéma un pur reflet du réel : « une certaine image du monde se construit sur l'écran, un projet politique se fait jour tendant vers un nouvel imaginaire » (Barlet, $1996: 54)$.

\section{Safi Faye et Mossane}

Safi Faye, qui écrit ses propres scénarios, précise au sujet de l'écriture dans le documentaire de Marie Mandy dont nous avons fait allusion :

\begin{abstract}
Moi, je travaille en fonction d'une idée qui me préoccupe. Voilà, une idée me préoccupe, m’empêche de dormir, m'empêche de penser à autre chose... Si... je fais à manger, ça il faut parce que toutes mes idées arrivent dans la cuisine, pendant que je fais à manger, la radio tourne, tout tourne et les phrases arrivent, donc tout papier qui traîne, j’écris dessus et même les chiffons j'écris dessus pour pas perdre l’idée parce que je pense... c'est très dur d'écrire. (Mandy, 2001).
\end{abstract}

Nous voudrions rappeler rapidement que Safi Faye est née en 1943 à Dakar et qu'elle a été institutrice au Sénégal durant sept ans. En 1970, elle décide d'entreprendre des études d'ethnologie et d'anthropologie à l’Université de la Sorbonne à Paris. Son objectif était, comme le signale le livret du film Mossane, de «mieux connaître l'Afrique ", avouant ainsi en quelque sorte une certaine méconnaissance de ce vaste territoire, tout en étant africaine. Cette formation se matérialise également par un intérêt manifeste pour le monde rural africain et pour la culture et les problèmes des populations d'Afrique noire. Parallèlement à son Doctorat, elle suit des cours de cinéma à l'École Supérieure Louis Lumière. Son 
travail a été amplement reconnu et récompensé. A titre d'exemple, on peut souligner que le Festival International des Films des Femmes de Créteil (AFFIF) lui a consacré une rétrospective en 1998.

Mossane, sorti en 1996, était présent au festival de Cannes de 1997 dans la sélection officielle de la section Un certain regard. Le film est tourné en wolof (sous-titré en français). L'auteure dit de son film qu'il s’agit d'un hommage à la femme africaine et elle précise que l'histoire retient trop souvent que la femme africaine est dominée : « C'est peut-être vrai en termes de pouvoir, mais ce sont les femmes qui élèvent leurs progénitures, fournissent la plus grosse part du revenu des familles et qui ont la plus forte influence sur la société. Elles portent la culotte. Mossane est l'une d'elles, vivant entre révolte et effacement » (Faye, 1996 : livret du film). En effet, se rebellant contre sa condition, Mossane brise le consensus social qui la détermine. Son « infidélité », aux dires de Barlet, « ouvre une telle brèche dans le jeu des intérêts en place que le groupe s’unit pour casser son énergie vitale et la faire rentrer dans le rang. Le village est alors confronté au drame que provoque son obstination : c’est dans la fuite ou dans la mort que la femme doit trouver refuge » (Barlet, 1996 : 117).

La trame du film tourne donc autour du thème du mariage forcé par intérêt, où la dot devient une arme de répression, d'interdiction de parole et de répression vis-à-vis de la femme. Dès sa naissance, les parents de Mossane l'ont promise en mariage à Diogoye, ayant émigré en France et voulant faire croire qu'il y a fait fortune. Cependant, Mossane est amoureuse de Fara, un jeune étudiant de retour au village pour les vacances. L'amour est réciproque. Pour mettre fin aux révoltes de Mossane, ses parents décident de célébrer le mariage par procuration dans la hâte et le plus grand faste. La belle Mossane prend la fuite mais elle meurt noyée en essayant d'échapper à un tragique destin qu'elle partagerait avec d'autres femmes d'histoire ou de légende, des vierges mythiques sacrifiées dans l'eau dans les mêmes circonstances.

Safi Faye a souvent recours à ce que Barlet appelle des «symboles en mouvement », une symbolique « de la vie ouvrant à une nouvelle façon d'agir » (Barlet, 1996 : 95) et montrant combien elle se sentirait enracinée dans la terre africaine, sorte de «matrice primordiale ». En effet, la lecture de Mossane, et c'est le cas pour d'autres films de la réalisatrice, ne peut « sous-estimer l'importance de ces éléments simples et évidents que sont notamment l’arbre, l'eau, la terre, l’air et le feu, dans ce qu'ils symbolisent d'une compréhension du monde, conférant au récit un sens profond » (Barlet, 1996 : 99). La réalisatrice explique que Mossane représente pour elle « une superbe créature inaccessible, âgée de 14 ans - dont les esprits, les êtres jeunes et vieux et la nature même tombent amoureux » (Barlet, 1997 : 10).

Dans mon conte, Mossane était de passage. Par contre, Magou Seck, l'actrice, existe. Elle a 21 ans. Son père étant décédé, je m’en occupe depuis. Elle était allée très peu à l'école avant le film. Actuellement, elle fréquente l'école de l'Alliance française à Dakar. Pouvoir maîtriser une autre langue autant que la sienne apporte un plus à l'individu. Se sentir à l'aise en s'exprimant en wolof, français, anglais, est admirable ! [...] L'acculturation est un enrichissement. [...] A 14 ans, la Mossane du film obéit à ses parents mais ressent les pulsions de l'adolescence. Point. Faire une fixation sur la tradition et la modernité seulement parce que Mossane est une Africaine est superflu. Mossane est une adolescente comme tout autre. Vouloir lui faire porter l'étiquette d'adolescente africaine serait aberrant. C'est l'âge où le corps, le visage, l'être changent à chaque instant. J'ai voulu capter ces images dans le film. (Barlet, 1997 : 10).

Or, la réalisatrice raconte, quelques années plus tard, à Marie Mandy que le film est une ode à la beauté et à la pureté des femmes de son pays : « J'ai voulu montrer que la plus belle femme du monde, la plus belle fille du monde était une Africaine. Je rêvais là-dessus. Elle ne peut avoir que 13 ans ou 13 ans et demi et j'ai fantasmé là-dedans et j'ai voulu qu'elle soit belle, pure, propre et tout ça appartient à mon continent, l’Afrique » (Mandy, 2001). En ce sens, le film montre ouvertement les corps nus des femmes et les pratiques et les techniques sexuelles transmises entre femmes, ce qui n'est que complémentaire :

Je pense que l'éducation sexuelle se fait avec les gens de sa génération. Ce n'est pas la mère qui inculque, c'est encore moins le père mais c'est souvent l'amie qui est un peu plus âgée ou même qui a le même âge mais qui s'est mariée avant qui transmet ce qu'elle a vécu. C'est normal. Si non où est-ce qu'on va apprendre à faire l'amour... à parler de sexualité. J'ai des amies qui me racontent ce qu'elles font avec leurs partenaires, je raconte ce que j'ai fait avec mes partenaires et on rigole. On ne peut pas le garder quand c'est bon. On le raconte à sa copine, même si elle n'utilise pas telle ou telle méthode pour faire ça, elle va essayer ce que l'autre a fait, ci et ça. (Mandy, 2001). 
La sexualité féminine et la sensualité des corps féminins est donc très présente dans le film : «quand c'est beau, je le montre. Oui, au Sénégal, quelqu'un m’a demandé de couper la « chevauchée » avant de projeter le film aux autorités... Ce qu'il appelle chevauchée, je l'ai appelé la sieste [...]. Quel mal y a-t-il à montrer une bonne sieste ? » (Barlet, 1997 : 10). Et elle ajoute : «peut-être ai-je osé montrer ce qui se fait la nuit et dont on ne parle pas le jour » (Barlet, 1997 : 10). Quant aux traits qui pourraient résulter d'une spécificité ou plutôt d'une perspective féminine, plus que sur la manière différente de filmer ou de raconter, nous voudrions insister sur des choix et des thématiques concrètes qui peuvent être mis en rapport avec d'autres discours de l'exclusion ou de la minorité et qui ont à voir avec la langue et avec la sexualité notamment, mais aussi avec ce positionnement politique déjà mentionné.

\title{
4. Cérémonies de l'eau et circulation du désir
}

En ce qui concerne la présence concrète de l'eau dans le film, celle-ci est assez significative. En effet, l'eau inonde le film, elle y est très présente. Nous allons approfondir notre analyse, en focalisant notre intérêt sur trois aspects : l'eau de la mer liée au paysage et à son symbolisme par rapport aux rites et aux croyances, l'eau douce et souterraine, en état naturel également, mais enfermée dans un puits lié à la femme, et l'eau utilisée dans des espaces intimes de bain entre femmes.

Le film commence avec Mossane dans l'eau de ce bras de mer, sorte de canal ou de détroit que l'on a identifié au Mama Nguedj, couverte d'une lumière dorée aveuglante en fort contraste avec sa silhouette noire. Elle y prend un bain à la fois ludique et rituel et se met à courir ensuite dans l'eau. Cet acte est accompagné de chants traditionnels sérères qui scandent le lavage du corps à moitié nu, évoquant les Pangool, les esprits des ancêtres qui prennent différentes formes, humaines ou naturelles, et qui intercèdent entre le monde des vivants et l'au-delà.

\begin{abstract}
A marée basse, quand Mossane se baigne / Dans les eaux de Mamangueth sur les rives du bras de mer / Les Pangools disparus en pleine jeunesse / Depuis la nuit des temps viennent contempler leur élue / Admirer leur favorite / Le mendiant errant poursuit ces génies de la savane / Qui, poussant d'amers soupirs, / Retournent à Sangomar ou l'embouchure gronde / Où les esprits habitent au sein des eaux mouvantes. (00:00-01:40)
\end{abstract}

L’empreinte de ces génies protecteurs est donc très visible dans la vie sociale et religieuse du village. Ils permettent une transcendance vers la divinité. Chacun a un pouvoir spécifique que les Sérères ont su réclamer grâce aux rituels souvent liés à l'eau car la sécheresse est l'une des pires malédictions dans ce coin de l’Afrique. Leur apparition au début du film près des eaux anticipe, en quelque sorte, la fin tragique de l'histoire avec le corps inerte de Mossane sur les rives de cette même lagune salée.

Ainsi, les habitants du village vénèrent l'eau sacrée qui les entoure hantée par les esprits. Guidés par le féticheur, tous s'acheminent vers la rive en procession, vêtus des meilleurs habits, chantant et jouant des instruments traditionnels pour lui faire des offrandes afin que la pluie revienne et que l'eau ne manque pas : « Ancêtre premier, jamais tu ne sombreras dans l’oubli. Ô Beep, notre divinité généreuse. Ô Nganiane qui coule sous ton ombre. Nous vous saluons. Accepte ce mil, nous te demandons de l'eau. Accepte ce riz, nous te demandons de la pluie. Accepte ce lait, nous te demandons de l'eau » (21:58-23:13). C'est aussi au bord de l'eau qu'ils vont sacrifier un bœuf blanc. Le sang mélangé à l'eau et à la terre crée une texture visuelle et un jeu chromatique très poétique et inquiétant en même temps : « Je demande pardon à Dieu pour ce couteau. Maïssa, nous t'offrons ce taureau. Nous souhaitons un bon hivernage. Qu'il pleuve à flot sur tout le pays » (23:50-25:03).

Le paysage est très présent dans le film surtout cette sorte de lagune salée à laquelle on a déjà fait allusion. Safi Faye filme de longs plans ouverts où elle montre la beauté de ce lieu magnifique. Les images où Mossane se promène avec Fara dans une barque au milieu des eaux et des petits îlots pleins de végétation sous fond de musique se mêlant au bruit de l'eau des vagues et des oiseaux marins sont vraiment poétiques, ainsi que leur promenade au bord de l'eau où l'on voit les barques des pêcheurs et où Mossane offre une conque à son amoureux toujours sous cette lumière dorée éblouissante qui fait ressortir les silhouettes noires (43:34-45:10) 
La famille de Mossane fête d'une manière cérémonieuse, en mangeant à la main un thiep délicieux, l'arrangement du mariage avec les parents de Diogoye. Dans ce rituel, la présence de l'eau pour le lavage des mains après le repas dans un grand bassin est aussi très significative car il tombe par terre et se casse lorsque la mère s'apprête à le retirer, ce qui signifie pour celle-ci un mauvais présage qui l'exaspère. Ce moment funeste à l'intérieur de la maison correspond avec l'arrivée d'un enfant mendiant qui s’approche de la maison pour demander à manger. C'est Mossane qui sort à l'extérieur et s'en occupe. Lorsqu'il reprend son chemin, les chants des femmes en voix off accompagnent la caméra qui le suit de dos : « Ô toi qui reprends la vie errante / Tu traverses les cours d'eau / Tu affrontes les bras de mer / Ô petit mendiant, où est ta mère ? / Ô Mendiant solitaire, quel chemin suis-tu ? / Mendiant errant de rive en rive, qui cherches-tu ? / Mendiant, dans la savane qui poursuis-tu ? » (28:57-29:32).

Mossane se révolte contre ce destin inéluctable et revendique sa liberté : « Dois-je faire mon choix ou suivre l’index pointé de ma mère ? [...] Je ne céderai pas, je choisirai. Ce que je vais te dire tu devrais le savoir : à la seule évocation de Fara, mon cœur bat comme un papillon dans une toile d'araignée. Quand je le vois mon cœur chavire. [...] Je refuse d’être l'hyène qui mange des cadavres » (1:06:40-1:07:40), dit Mossane à Samba le « fidèle griot » envoyé par Mingué, la mère, pour la convaincre. Courageuse, elle affronte sa mère directement : « mon mariage ça me regarde. J’épouserai celui que j’aime, Fara » (1:07:51-1:08:09), mais Mingué finira par la frapper au visage (« qui ça ? Jamais de la vie ») et le père lui jettera un bout de bois à la tête : «Tu nous exaspères. Ne prononce plus tes paroles de diablesse chez moi ! (1:08:101:18:18). On voit ensuite Mossane creusant la terre, toute éplorée et désespérée : « Je te maudis, terre stérile. Tu ne sers à rien. Pangools mes ancêtres, je me sens seule ici-bas » (1:08:24-1:08:58).

L'eau est aussi présente dans un puits proche au village qui apparaît à deux reprises, le jour et la nuit. Lorsque Mossane, comme d'autres jeunes femmes du village, va chercher ce bien précieux, on voit son image reflétée dans l'eau emprisonnée, une image qui est brisée et défigurée par le seau qu'elle y jette pour faire monter l'eau produisant un sourd bruit, ce qui anticipe encore une fois la fin funeste du personnage en quête d'émancipation. Seule Mam, sa grand-mère, l'aide à poser le grand récipient sur sa tête pour rapporter l'eau à la maison dans un geste de complicité et de compréhension entre elles : «c'est inacceptable ! Nous allons nous battre » (1:00:43); ou encore : « Quand Mossane n’est pas joyeuse, je suis triste. Quand elle pleure, je souffre (1:10:10-1:10:25). Après la cérémonie des noces forcées, Mossane s'échappe au beau milieu de la nuit. Dans sa course effrénée où l'on n’entend que le bruit des animaux nocturnes, ses pas accélérés et sa respiration agitée, comme s’il s’agissait d'un animal traqué et menacé, elle crie « au secours » et s’arrête devant le puits où l'eau noire se confond avec la nuit néfaste et le visage de Mossane inondé par la sueur. Seule la lune laisse apercevoir son flou reflet au fond du puits. Dans une barque, tel un spectre errant au milieu des eaux dans l'obscurité la plus complète où seuls ses habits blancs contrastent avec le noir des ténèbres, elle tâche de traverser cette lagune sombre, rappelant clairement le passage symbolique au royaume des morts. Apeurée par les sons de l'eau et des créatures de la nuit et emportée par l'angoisse et la détresse, elle n'aura qu'à crier et à se coucher dans cette barque-berceau-cercueil qui l'emmènera vers son tragique destin. En effet, elle sera retrouvée noyée le lendemain sur les rives de ce bras de mer qui l'a attrapée pour toujours et l’a fait, également, entrer dans le mythe.

Le film est également marqué par des plans généraux extérieurs ou intérieurs où l'on voit le travail des femmes et leur importance pour la communauté : au marché ou en réalisant des tâches ménagères comme faire la vaisselle ou préparer à manger. La réalisatrice aime aussi à placer la caméra derrière le dos des personnages ou à travers des jalousies ou des éléments qui tamisent le regard et qui créent un jeu d'ombre et de lumière. Bien qu'elle fixe clairement le regard sur la protagoniste, elle n'adopte pas son point de vue, prétendant un regard « objectif » ou extérieur, qui met en relief beaucoup d'éléments naturels (arbres, animaux, plantes, terre etc.), sociaux et anthropologiques, à la manière d'un documentaire.

L'eau est également présente dans les rituels intimes entre femmes. À l'heure de la sieste, Dibor fait l'amour avec son mari Daouda. L'arrivée inattendue de Mossane interrompt la scène au moment le plus intense. Alors les deux amies se livrent à un exercice de nettoyage des corps, à la fois complice et érotique, dans une salle de bains improvisée derrière un enclos de paille et de roseaux secs. La caméra s'attarde et se promène sur les corps nus, le sexe toujours couvert par un pagne, sur les dos, sur les cheveux et les visages, pour s'arrêter sur les pieds et la mare d'eau qui se forme par terre. Les deux jeunes femmes, dans une sororité complice, se savonnent entre elles, se jetant ensuite de l'eau sur la tête dans un jeu enfantin et sensuel : «Quand Daouda revient des champs, rompu par le travail et que je porte mon pagne d'amour, il retrouve toute sa vigueur. Et quand je le chevauche, mon cœur saute comme une baguette sur un tam-tam. Je vais me doucher. Mets ce pagne »(30:42-31:58). Après avoir partagé des rires francs et un beau moment d'amitié dans cette douche improvisée, elles rentrent à la maison de Dibor où elles continuent leur toilette à moitié nues, donnant suite au 
séchage des peaux à l'aide de sensuels frottements, tout en reprenant cette leçon sur les pratiques amoureuses dans une initiation à la fois innocente et directe.

Mossane : Dis-moi. Comment m’y prendre avec Fara sans arriver au pire ?

Dibor : Ma chère, les amants ça existe ! Fara peut te frotter du bout de son sexe, doucettement, et de ton clitoris va monter le plaisir.

Mossane : De quoi tu parles?

Dibor : L'important c'est d'être prudents, toi surtout. Interdis-lui une seule chose : de « tomber dans ton fruit de mer ».

(31:59-32:33).

Il y aura un autre moment intime de nettoyage à l'extérieur, plus bref, entre Mossane et sa mère mais celui-ci sera plutôt marqué par la méfiance et la hiérarchie qui s'établit entre elles. En effet, la communication est presque absente, Mossane ne se déshabille pas et elle ne fait qu'obéir à sa mère qui l'utilise comme une servante : « Mossane. Viens me frotter le dos. Ça suffit merci » (36:49-37:28). En tout cas, c'est une belle excuse pour la réalisatrice qui montre le corps nu de cette femme aux formes généreuses. Elle fixe encore une fois la caméra sur de très beaux détails de la coiffure et du dos mouillé et savonné de la mère où se projettent de capricieux dessins produits par la mousse blanche sur le corps noir et par la lumière qui s'infiltre à travers la grille de paille censée protéger cette nudité du regard extérieur mais s'ouvrant impudiquement à nous yeux. Safi Faye insiste beaucoup sur le visage des femmes, sur les cheveux et leur parure, ainsi que sur des parties du corps, comme le dos, l'épaule ou les pieds, qui sont peu montrées en général. Même si elle montre la femme comme faisant partie de la vie de la communauté dans les rituels et les rythmes de la vie quotidienne, il est évident que l'œil de la caméra se fait aussi solidaire de la « sororité » qui lie ces femmes, bien que la mère, sorte de rivale, soit exclue de cette complicité fusionnelle.

\section{En guise de conclusion}

Nous pourrions avancer, pour conclure, que Mossane est un film où l'eau et le désir sont très présents. Il s'agit d'une narration riche en éléments qui font de ce conte poétique et social un « chant d'amour » (pour reprendre le titre de Jean Genet) dédié à la femme (africaine, en l’occurrence), symboliquement piégée dans un entre-deux, mais qui montre ouvertement son courage et son désir d'émancipation. Même si nous ne l'avons pas abordé de ce point de vue, il faut mettre en relief que Safi Faye, grâce à son particulier regard ethnographique, a voulu aussi rendre hommage au peuple sérère auquel elle appartient en montrant sa culture et ses rites avec finesse mais portant également un regard critique sur certaines traditions qui emprisonnent la femme. En effet, le film mélange un ton général très mélancolique, voire tragique et prémonitoire, accentué par le symbolisme de l'eau, mais nuancé par de petites touches humoristiques grâce auxquelles la réalisatrice nous fait découvrir les dessous du rituel matrimonial, ou comment le fiancé finit par «acheter» l'ensemble des villageois avec l'argent. Dans ce film, Safi Faye fait donc une vaste place à l'éducation des femmes (africaines) et à la résistance des jeunes générations en sublimant leurs aspirations de liberté et d'indépendance. Le tout habilement porté par une musique très présente dans le film, choisie non sans humour également et regorgeant de couleurs chaudes et vives où tous les détails ressortent, surtout dans les habits et les objets, des images magnifiques signées par Jürgen Jürges, cameraman fidèle, entre autres, de Fassbinder. La cinéaste a fait également preuve de courage et esprit d'indépendance pour mener à terme son projet car toutes ces belles images tournées en 1990 n’ont pu être montées avant 1996, «après une série de désaccords financiers et juridiques avec son producteur français ». Le film a finalement été produit et diffusé en Suisse par Trigon-film (http://www.trigon-film.org/fr/movies/Mossane).

Nous voudrions finir avec les mots de Barlet qui souligne que « marquées par le statut d'infériorité que leur imposent nos sociétés patriarcales, les femmes savent souvent mieux que les hommes se mettre à la place de l'Autre et ne pas penser automatiquement que l'Autre pense comme elles ». La femme est « celle qui se soulève, qui refuse un ordre établi qui la réduit ». Et il ajoute que Mossane a « la maturité d'une femme cinéaste qui sait puiser dans sa vitalité un regard d'enfance. Même s'il rappelle avec mélancolie que le bonheur n'est pas de ce monde, ce film est un vigoureux et touchant appel contre les immobilismes, une affirmation de vie » (Barlet, $1997: 9$ ). 


\section{Références bibliographiques}

BARLET, Olivier (1996). Les Cinémas d'Afrique noire. Le regard en question. Paris : L'Harmattan.

BARLET, Olivier (1997). «Entretien avec Safi Faye » dans Africultures. Les Africaines, nº 2, 8-11.

BAYON, Estelle (2007). Le Cinéma obscène. Paris : L’Harmattan.

Benhamou, Anne-Françoise (2007). "Metteuses en scène : le théâtre a-t-il un genre ? » en OutreScène $\mathrm{n}^{\circ}$ 9. Théâtre National de Strasbourg : 4-6.

FAYE, Safi (1996). Mossane. Dakar : Muss Cinématographie.

GARDIES, André et HAFFNER, Pierre (1987). Regards sur le cinéma négro-africain. Bruxelles : OCID.

MANDY, Marie (2001). Filmer le désir (voyage à travers le cinéma des femmes). Bruxelles : Saga Films.

Pujante Gonzalez, Domingo (2013). «De la pertinence de l’obscène au féminin au tournant du XXI ${ }^{\mathrm{e}}$ siècle » dans Ob/scena. L'obscène au féminin au tournant du XXI ${ }^{e}$ siècle. Paris : L'improviste.

SEgARRA, Marta (2010). Nouvelles romancières francophones du Maghreb. Paris : Karthala. 